\title{
Editorial
}

\section{Is Type II (non-insulin-dependent) diabetes mellitus not so "genetic" after all?}

\section{J. L.Hopper}

The University of Melbourne, Australian NHMRC Twin Registry, 200 Berkeley Street, Carlton, Victoria 3053, Australia

Early clinic-based studies of volunteer twins claimed to find a high concordance for diabetes in identical twins and interpreted this as evidence of a strong genetic basis for the disease. This was despite the wellknown biases of those non-population-based studies and the strong evidence of lifestyle-related risk factors. There is also a reduced tendency for the involvement of genetic factors for later onset cases of a disease, as for example in breast cancer. There is substantial evidence for a strong genetic basis of insulin dependent diabetes mellitus, as supported by a recent Danish twin study [1].

If conducted and analysed properly, studies of the similarities and differences within both monozygotic (MZ) and dizygotic (DZ) twin pairs can be used to make guarded inference about the roles of genetic and non-genetic factors on the aetiology of disease. Twin studies can never prove the existence of a genetic susceptibility, however, and at best can indicate the extent of genetic determination consistent with a specific model, under certain important assumptions [2].

In this issue of the journal the question of a genetic aetiology of Type II (non-insulin-dependent) diabetes mellitus is addressed by two new twin studies. On face value, the two studies appear to be giving contradictory messages. The population-based Danish study [3] suggests that while $\mathrm{MZ}$ pairs are concordant for diabetes, and more so than DZ pairs, the difference is not large and consistent with chance. The probability that one twin of an MZ pair is diabetic given that the other has diabetes was estimated to be $50 \%(95 \% \mathrm{Cl} 32-71 \%)$, and they called this "probandwise concordance". For DZ pairs this estimate was $37 \%(19-55 \%)$, and the two estimates were not nominally different at the 0.05 level of significance. On the other hand, the clinic-based study in the United Kingdom [4] suggests that if one twin of an MZ pair is diabetic, the probability that the non-diabetic twin will become diabetic within the next 15 years is as high as $76 \%(58-91 \%)$. They used the term "pairwise concordance rate". But like twin pairs these two articles have similarities and differences. The challenge in both instances is to understand the reasons.

\section{Population versus clinic-based studies}

Perhaps the most important difference between the studies is in the sampling. The strength of the Danish study is the use of national registration to define twins within a given age range living in Denmark. The UK study was clinic-based, and as the authors state, apparently subjects in such studies are likely to have more severe diabetes. Without having a clearly defined sampling population it is not possible to make statistical inference with any confidence.

\section{Definition of diabetes}

The clinic-based study uses effectively two different definitions of diabetes. For the first affected twin the definition is by diagnosis and referral to the British Diabetic Twin Study. For the second affected twin diabetes was confirmed when for the first time certain standard criteria were reached following an annual clinical test. Therefore, there is a greater level of scrutiny of the twin who was non-diabetic at enrolment of the affected twin. For the population-based study the same definition of diabetes applies to each twin of the pair, and is done at the same time.

\section{Measures of concordance}

There has been a great deal of confusion in the literature about the concept of twin concordance as these 
two papers further illustrate. Firstly, these concordances aren't "rates"; there is no time component and they are simply proportions. Secondly, concordance refers to a cross-sectional concept of sampling pairs from a population.

Two measures of concordance are typically used, pairwise and casewise [5]. Both refer to conditional probabilities. The former refers to the probability that both members are affected, given that at least one member of the pair is affected. That is, $\mathrm{P}_{\mathrm{p}}=\mathrm{P}\left(\mathrm{Y}_{1}=1\right.$ and $\mathrm{Y}_{2}=1 \mid \mathrm{Y}_{1}=1$ or $\left.\mathrm{Y}_{2}=1\right)$, where $Y_{1}$ and $Y_{2}$ refer to the disease state of twins 1 and 2 of a pair, which is 1 , if affected, and 0 , if not. The latter is based on a case, and is $\mathrm{P}_{\mathrm{c}}=\mathrm{P}\left(\mathrm{Y}_{2}=1 \mid \mathrm{Y}_{1}=1\right)$. A third "concordance (rate)", the probandwise concordance, is mentioned in the literature and by Poulsen et al. [3] but it is not a measure of concordance (like casewise or pairwise concordance), but an estimator of concordance. It is used when only pairs in which at least one twin is affected are sampled. The distinction between an estimator and the parameter it is designed to estimate has often been obscured in part due to poor notation [6].

The situation is complicated even further because historically the term probandwise concordance has been used in two ways. Firstly, as in [3], it is defined as $2 \mathrm{n}_{11} /\left(2 \mathrm{n}_{11}+\mathrm{n}_{\mathrm{d}}\right)$, where $\mathrm{n}_{11}$ refers to the number of pairs in which both twins are affected (i.e. $Y_{1}=1$ and $Y_{2}=1$ ), and $n_{d}$ refers to the number of disease discordant pairs (i.e. $\mathrm{Y}_{1}=1$ and $\mathrm{Y}_{2}=0$, or vice versa). If there is "complete ascertainment", in a sense that will be discussed below, this is the maximum likelihood estimate of the casewise concordance [5].

The "ascertainment probability", $\pi$, is the probability that an affected twin will be identified from the original sampling of the population. If $\pi$ is close to one, ascertainment is considered to be almost "complete", and the formula above gives a good estimate of the casewise concordance. To estimate $\pi$ we need to distinguish between pairs who were identified as being concordant for disease only due to the second, clinical stage of sampling. These are referred to as "singly" ascertained and their number is $\mathrm{n}_{11 \mathrm{~s}}$. Disease concordant pairs according to both the questionnaire and the clinical investigation are called "doubly" ascertained, and their number is $\mathrm{n}_{11 \mathrm{D}}$. The ascertainment probability is then estimated by $\Pi=2 \mathrm{n}_{11 \mathrm{D}} /$ $\left(2 \mathrm{n}_{11 \mathrm{D}}+\mathrm{n}_{11 \mathrm{~S}}\right)[5]$.

Consider the two-stage sampling of the Danish study. Among the 303 pairs who were clinically examined (125 MZ, $178 \mathrm{DZ})$, the prevalence of self-reported Type II diabetes was $5.9 \%$, equivalent to 36 affected patients. Pairwise concordance for self-report was 0.25 in $\mathrm{MZ}$, and 0.0 in $\mathrm{DZ}$ pairs, so all $\mathrm{DZ}$ pairs were discordant on self-report. On examination 79 were found to have Type II diabetes by the oral glucose tolerance test. Therefore, 43 affected twins were newly identified. All eight DZ concordant pairs were identified by the second stage, comprising 16 patients, so $n_{11 \mathrm{~S}}$ is at least 8 . At most six of the MZ pairs were concordant on self-report, so $n_{11 \mathrm{D}}$ is less than 6 . Hence $\Pi$ can be at most 0.6 , suggesting incomplete ascertainment.

To adjust for this, one must use the probandwise estimator of the casewise concordance which is $\left(2 n_{11 D}+n_{11 S}\right) /\left(2 n_{11 D}+n_{11 S}+n_{d}\right)[5,6]$. For DZ pairs, $\mathrm{n}_{11 \mathrm{D}}=0$, so the casewise concordance is estimated to be 0.23 . For MZ pairs it is less than 0.45 . That is, the difference between casewise concordance is still small and a formal test of significance would still show no evidence for a difference between $\mathrm{MZ}$ and DZ pairs.

As the UK study does not involve sampling from a defined population and there is no information about the number of doubly or singly ascertained disease concordant pairs, this exercise cannot be carried out. Hence its title is a misnomer.

\section{Just what is heritability?}

Poulsen estimated the "heritability" of diabetes from their twin data to be $26 \%$, although it was not statistically significant and had a very large confidence interval. What does heritability mean in this context? It is not the proportion of diabetes attributable to genetic factors [7], as one might intuitively anticipate. Rather, it is a much more complicated, and even fanciful, concept! To derive this number, one must accept that everyone has a "liability" to diabetes and this variable has a normal distribution across the population, but it has not been measured. The probability that a person is diabetic is presumed to depend on his liability. From the pattern of concordance and discordance for disease in twin pairs the correlation between twins in their unmeasured liabilities is estimated. Then, a simple linear model for the effects of genetic and environmental causes of variation in liability is assumed. Heritability is defined to be "the proportion of variation in liability across the population that the model attributes to genetic factors", and is estimated from the twin data. The problem is that the normal distribution assumption cannot be tested.

Given that one believes in the existence of a normally distributed liability, however, the extent to which shared environmental factors explain population variation in liability can estimated. Poulsen et al. found this to be $41 \%$ or $60 \%$, depending on whether a genetic effect is included in the model. The bottom line is that, with these numbers of affected twin pairs studied, there is not enough data to resolve which of these components best describes the data. Because it has yet to study DZ pairs, the clinicbased study cannot make any claims about genetic or environmental determinants; the concordance between MZ pairs could be due to shared genes or shared environment. 
Resolution of these "nature versus nurture" issues might come by long term follow-up of the Danish, population-based cohort of MZ and DZ twin pairs. The clinic-based study is essentially a cohort study and could be used to assess the determinants of time to diabetes in the originally non-diabetic twin. It would have been informative to know what characteristics, if any, could distinguish the twins who became diabetic earlier from those who did so later, or those who had not yet become diabetic. Perhaps this issue will be addressed in future studies from these groups.

\section{References}

1. Kyvik KO, Green A, Beck-Nielsen H (1995) Concordance rates of insulin dependent diabetes mellitus: a populationbased study of young Danish twins. BMJ 311: 913-917

2. Hopper JL (1996) Commentary: Genes for osteoarthritis: interpreting twin data. BMJ 312: 943-944

3. Poulsen P, Kyvik KO, Vaag A, Beck-Nielsen H (1999) Heritability of non-insulin-dependent diabetes mellitus (NIDDM) and abnormal glucose tolerance - a populationbased twin study. Diabetologia

4. Medici F, Hawa M, Ianari A, Pyke DA, Leslie RDG (1999) Concordance rate for NIDDM in monozygotic twins: actuarial analysis. Diabetologia

5. Hopper JL (1998) Twin concordance. In: Encyclopedia of Biostatistics. Wiley, London, Vol. 6, pp 4626-4629

6. Witte JS, Carlin JB, Hopper JL (1999) A likelihood approach to estimating twin concordance for dichotomous traits. Genetic Epidemiology (in press)

7. Hopper JL (1998) Heritability. In: Encyclopedia of Biostatistics. John Wiley \& Sons, London, Vol. 3, pp 1905-1906 